encapsulation around the material. A protein-resistant, liquidto-solid curing material could have expanded use as anon-fouling, protein-resistant coating for a variety of metal-based implants.

Materials and Methods The resistance to protein adsorption is quantified via protein depletion from the blood, as well as through analysis of desorbed proteins from sample surfaces via Tween-20. Detection and quantification of proteins is performed via PPODA-QT samples $(n=12)$ were prepared and cured in $4 \mathrm{~mm}$ diameter cylindrical molds with a height of $10 \mathrm{~mm}$. PPODA-QT samples were each immersed in $1.5 \mathrm{~mL}$ of heparinized rabbit whole blood within a $2 \mathrm{~mL}$ polypropylene vial. Vials were placed on a shaker plate for15 minutes to ensure maximal interfacing between blood and the samples. With the majority of protein adsorption happening within seconds, 15 minutes is sufficient for protein adsorption. Positive controls $(n=4)$ were created by preparing $4 \mathrm{~mm}$ diameter and $10 \mathrm{~mm}$ thick polyurethane cylinders and subjecting them to the same blood immersion procedure. Negative controls $(n=4)$ were created by filling vials with blood and no sample to give a baseline level for protein adsorption onto the vials themselves. Proteins will be identified and quantified via label-free spectroscopy techniques.

Results PPODA-QT has been shown to exhibit exemplary protein-resistant properties as well as minimal encapsulation and inflammatory response when implanted while providing a relatively uniform surface for neointimal tissue growth across the device at the neck of the aneurysm. Comparison of PPODAQT to the positive controls results in statistically significant reduction of protein depletion from blood samples. Verification of this result via analysis of desorbed proteins is underway.

Conclusion The protein-resistance of PPODA-QT as shown in this study makes it an interesting material candidate for a variety of surgical applications. A liquid-to-solid curing material with inherent protein-resistant properties could be utilized not only as a novel liquid embolic for treatment of intracranial aneurysms and AVMs, but could also be used as a nonfouling, bioinert coating for metallic implants such as stents, flow diverters, and coils.

Disclosures W. Merritt: 1; C; Northern Arizona University, Aneuvas Technologies, Inc.. 5; C; Aneuvas Technologies, Inc. A. Ducruet: 1; C; Northern Arizona University, Aneuvas Technologies, Inc. T. Becker: 1; C; Northern Arizona University, Aneuvas Technologies, Inc.. 4; C; Aneuvas Technologies, Inc.. 5; C; Aneuvas Technologies, Inc.

\section{P-036 SENSITIVITY OF THE RACW SCORE IN THE DETECTION OF LARGE VESSEL OCCLUSIONS DURING WORKING AND NON-WORKING HOURS}

${ }^{1} E$ Hitomi, ${ }^{1} \mathrm{M}$ Jumaa, ${ }^{1} \mathrm{~S}$ Zaidi, ${ }^{1} \mathrm{~J}$ Shawver, ${ }^{1} \mathrm{~A}$ Korsnack, ${ }^{1} \mathrm{~A}$ Castonguay, ${ }^{1} \mathrm{R}$ Burgess, ${ }^{1} \mathrm{~V}$ Kung, ${ }^{1,2} \mathrm{H}$ Salahuddin*. ${ }^{1}$ Neurology, University of Toledo, Toledo, OH; ${ }^{2}$ Neurology, Vascular Neurology of Southern California, Thousand Oaks, CA

\subsection{6/neurintsurg-2021-SNIS.72}

Introduction Multiple studies have shown that faster treatment times for ischemic strokes result in improved clinical outcomes. Pre-hospital triage scores aim to identify large vessel occlusions in the field and allow earlier activation of stroke teams in the hospital.

Objective To compare the sensitivity of the pre-hospital Rapid Arterial oCclusion Evaluation (RACE) score for the detection of large vessel occlusions during working hours and non-working hours.

Methods We retrospectively reviewed all patients presenting with a RACE score of $\geq 5$ to one comprehensive and one thrombectomy capable hospital between July 2015 and December 2019. Baseline demographics, time of hospital arrival, presenting NIHSS score, intravenous tPA and mechanical thrombectomy metrics, ninety day modified Rankin scores, discharge disposition, and final discharge diagnosis were recorded. Patients presenting between 7 AM to 6 PM during weekdays were considered to present during 'Working hours' whereas patients presenting between $6 \mathrm{PM}-7 \mathrm{AM}$ on weekdays or anytime during weekends were considered to present during 'Non-working hours'. The primary outcome of interest was diagnosis of large vessel occlusion. Secondary outcomes included diagnosis of neurovascular event, discharge diagnosis, and good clinical outcome defined as ninety day modified Rankin Scale (mRS) of $\leq 2$.

Results Over a 4.5 year period, this study analyzed 687 patients who presented to the hospital via Emergency Medical Services with a RACE score of 5 or more. The average age of the cohort was 71.4 years and women comprised $55 \%$ of the cohort. Median NIHSS was comparable in the Working (13) and Non-working $(14 ; \mathrm{p}=0.48)$ groups. Intravenous tPA administration $(21.7 \%$ vs. $26.5 \% ; \mathrm{p}=0.15)$ and risk factors including hypertension, diabetes mellitus, previous stroke, and prevalence of atrial fibrillation were comparable between the two groups. There was no significant difference in the diagnosis of large vessel occlusion (36.4\% vs $34.6 \%$ ) or final discharge diagnosis. Sensitivity of the RACE score for detection of neurovascular events (TIA, ischemic stroke, intracranial hemorrhage) was improved during non-working hours $(75.1 \%)$ compared to working hours $(67.2 \% ; \mathrm{p}=0.02)$. Although door to groin puncture \& recanalization times were shorter during working hours, there was no significant difference in the rate of good clinical outcomes $(54.1 \%$ vs. $51.5 \% ; \mathrm{p}=0.76)$ in patients undergoing mechanical thrombectomy.

Conclusion The sensitivity of the RACE score for detecting large vessel occlusions does not vary significantly during working and non-working hours. However, patients who present with high RACE scores during working hours are more likely to have a diagnosis of a stroke mimic than those presenting off hours.

Disclosures E. Hitomi: None. M. Jumaa: None. S. Zaidi: None. J. Shawver: None. A. Korsnack: None. A. Castonguay: None. R. Burgess: None. V. Kung: None. H. Salahuddin: None.

\section{P-037 COMPARISON OF RADIATION EXPOSURE AND CLINICAL OUTCOMES BETWEEN TRANSRADIAL AND TRANSFEMORAL DIAGNOSTIC CEREBRAL APPROACHES: A RETROSPECTIVE STUDY}

${ }^{1,2} \mathrm{C}$ Amankwah*, ${ }^{1,2} \mathrm{~L}$ Lombardo, ${ }^{1} \mathrm{~J}$ Rutledge, ${ }^{1,2} \mathrm{~A}$ Sattar, ${ }^{1,2} \mathrm{~B}$ Chancellor, ${ }^{1,2} \mathrm{H}$ Moyle,
${ }^{1,2} \mathrm{D}$ Altschul. 'Neuroscience, Valley Health System, Ridgewood, NJ; ${ }^{2}$ Neurosurgeons of New
Jersey, Ridgewood, NJ

10.1136/neurintsurg-2021-SNIS.73

Background Transradial (TRA) catheterization for neuroendovascular procedures is effective and associated with fewer complications than transfemoral (TFA) procedures. However, 